\title{
Fatigue Behavior of Non-Optimized Laser-Cut Medical Grade Ti-6Al-4V-ELI Sheets and the Effects of Mechanical Post-Processing
}

\author{
André Reck ${ }^{1, *} \mathbb{0}$, André Till Zeuner ${ }^{1}$ and Martina Zimmermann ${ }^{1,2}$ \\ 1 Institute of Materials Science, Technical University of Dresden, 01062 Dresden, Germany \\ 2 Department of Materials Characterization and Testing, Fraunhofer-Institute for Material and Beam \\ Technology IWS, 01277 Dresden, Germany \\ * Correspondence: andre.reck@tu-dresden.de
}

Received: 18 June 2019; Accepted: 26 July 2019; Published: 30 July 2019

\begin{abstract}
The study presented investigates the fatigue strength of the $(\alpha+\beta)$ Ti-6Al-4V-ELI titanium alloy processed by laser cutting with and without mechanical post-processing. The surface quality and possible notch effects as a consequence of non-optimized intermediate cutting parameters are characterized and evaluated. The microstructural changes in the heat-affected zone (HAZ) are documented in detail and compared to samples with a mechanically post-processed (barrel grinding, mechanical polishing) surface condition. The obtained results show a significant increase $(\approx 50 \%)$ in fatigue strength due to mechanical post-processing correlating with decreased surface roughness and minimized notch effects when compared to the surface quality of the non-optimized laser cutting. The martensitic $\alpha^{\prime}$-phase is detected in the HAZ with the formation of distinctive zones compared to the initial equiaxial $\alpha+\beta$ microstructure. The HAZ could be removed up to $50 \%$ by means of barrel grinding and up to $100 \%$ through mechanical polishing. A fracture analysis revealed that the fatigue cracks always initiate on the laser-cut edges in the as-cut surface condition, which could be assigned to an irregular macro and micro-notch relief. However, the typical characteristics of the non-optimized laser cutting process (melting drops and significant higher surface roughness) lead to early fatigue failure. The fatigue cracks solely started from the micro-notches of the surface relief and not from the dross. As a consequence, the fatigue properties are dominated by these notches, which lead to significant scatter, as well as decreased fatigue strength compared to the surface conditions with mechanical finishing and better surface quality. With optimized laser-cutting conditions, HAZ will be minimized, and surface roughness strongly decreased, which will lead to significantly improved fatigue strength.
\end{abstract}

Keywords: Titanium alloys; Ti-6Al-4V-ELI; fatigue; laser cutting; post-processing; $\alpha^{\prime}$-martensite; HAZ; barrel grinding; notch; fracture

\section{Introduction}

Titanium alloys are a frequently used material in industrial applications with ongoing market growth in recent years [1]. The industrial applications involve a wide spectrum from the aerospace and automobile sector, the chemical industry, to the field of medical engineering, such as applications in osteosynthesis. The reasons for this broad range of applications are high specific strength even at higher temperatures, excellent corrosion resistance, and the ability to adjust the material properties to a great extent with the optimization of the microstructure, as well as surface properties [1-3].

Titanium alloys possess high sensitivity to processing conditions and the subsequent fatigue loading during the application, strongly depending on the chemical composition, initial microstructure, 
and thermomechanical history [1,4-6]. Insufficient understanding or negligence of these factors may, therefore, result in an under- or overestimation of the fatigue strength and, as a consequence, either the failure to explore the full potential of titanium alloys or the leading to fatal failure cases.

The processing method of laser-cutting is a frequently used technique to transfer the sheet pre-product to the end geometry for the application. The high cost-effectiveness, economic efficiency, variability, as well as the possibility to produce complex geometries in a short time, are only a few of the many advantages [7-9]. However, the thermal input due to the laser must be adjusted and optimized regarding the surface roughness and resulting heat-affected zone (HAZ). Otherwise, microand macro-notch effects can negatively affect the fatigue behavior leading to catastrophic pre-mature failure in their application [4,10-12]. The local changes of the microstructure in the HAZ may also have a significant influence on the mechanical properties and on the fatigue strength, in particular [4,13-15]. However, the mechanical post-processing or finishing methods can improve the surface quality after laser-cutting or related processing methods in a significant way, which was proven in several studies $[9,10,16-18]$. The effects of surface roughness and the influence of the HAZ of a laser-cut component are, nevertheless, the subject of current research. Depending on the extent of strength reduction, the laser-cutting process has to be adjusted so as to form a favorable surface quality at the expense of the processing speed, while costly post-processing of the surface to remove the HAZ also has to be discussed with regard to its effectiveness on fatigue strength improvement.

The $(\alpha+\beta)$ alloy Ti-6Al-4V-ELI, which is analyzed in the present study, is one of the standard alloys in the medical sector due to its exceptional combination of high specific strength, good ductility, and remarkable corrosion resistance. As sheet metal, this alloy is mostly processed by laser-cutting and mechanically post-processed with barrel or vibratory grinding methods. While detailed investigations on the surface quality and the HAZ due to laser-cutting and their consequence with regard to the fatigue properties are limited, it is of a common consensus that from a general point of view, a significant negative influence of increasing surface roughness on fatigue behavior is expected [10,14-16,19]. Studies on the general interaction of laser and material surface concentrate on the temperature field, kerf development, and parameter studies (type of laser, power, speed, etc.) [13,20-24]. Fatigue properties of Ti-6Al-4V-ELI have been investigated intensively in the past [10,14-17,25-29]. The sensitivity against surface roughness and underlying surface near microstructure is assessed and confirmed. Da Silva et al. [10] demonstrated a theoretical and experimental decrease in fatigue strength with increasing surface roughness. Morita et al. [27] focused on the influence of short term aging to improve the fatigue performance of notched Ti-6Al-4V-ELI. The development of $\alpha^{\prime}$-martensite phase during quenching led to a retardation of crack propagation and, therefore, better fatigue properties [27]. The mechanical post-processing methods to improve the fatigue properties of Ti-6Al-4V-ELI were the subject of investigations, as was the influence of the environment, which is especially important for application in the human body $[10,16,17,30,31]$. However, to the best of the author's knowledge, no direct studies are found focusing on the interaction between laser-cutting, the subsequent mechanical post-processing, and the endurable stress amplitudes. Furthermore, it is known from related studies concerning laser welding and comparable methods to which extent the laser can change the local microstructure, and therefore, the mechanical and fatigue properties [32-34].

Therefore, the study aims to identify the principle changes that are obtained regarding surface quality and near-surface microstructure due to laser-cutting with intermediate (non-optimized) cutting parameters and to evaluate these effects with respect to the fatigue behavior of Ti-6AL-4V-ELI. The possible local changes in the HAZ-microstructure shall be clarified, and the resulting surface quality assessed. Furthermore, the fatigue strength of the specimens after mechanical post-processing with the method of barrel grinding will be compared to samples with an as-cut surface and with a surface after manual mechanical polishing. Surface roughness is also investigated in conjunction with the crack initiation sites after fatigue loading. 


\section{Materials and Methods}

The studied medical grade Ti-6Al-4V-ELI (ISO 5832-3) sheet pre-product was purchased from the supplier MetSuisse Distribution AG (Zug, Switzerland) and originally produced by RTI International Metals Inc. (Pittsburgh, PA, USA). The sheet thickness was $0.8 \mathrm{~mm}$ and the laser-cutting process was realized with a disk laser (TruDisk 5001-Fa. TRUMPF, Ditzingen, Germany) with $3 \mathrm{~kW}$ laser power at $25 \mathrm{~m} / \mathrm{min}$ cutting speed. To minimize the possible chemical reactions due to oxygen or nitrogen, which can lead to hard and brittle $\mathrm{TiO}_{2}$ or TiN surface layers, laser-cutting was carried out under argon atmosphere with an argon pressure of 6 bar. All laser cutting parameters (Table 1) were chosen to display an average parameter set for the Ti-6Al-4V-ELI alloy. Since the process optimization was not foreseen in the scope of the study presented, the parameters of the laser-cutting process were chosen on the basis of practical knowledge and represent an intermediate condition; however, allowing a general analysis of the possible changes in the microstructure and the extent of surface roughness effects on the fatigue behavior. The geometry used for the laser-cut fatigue samples was developed in previous studies on medical implant alloys [12] and is depicted in Figure 1.

Table 1. Laser cutting parameters applied for Ti-6l-4V-ELI sheets on the TruDisk 5001 laser (Fa. TRUMPF-Series Tru Laser 7025).

\begin{tabular}{cccccccc}
\hline $\begin{array}{c}\text { Cutting } \\
\text { Parameters }\end{array}$ & Laser Power & $\begin{array}{c}\text { Cutting } \\
\text { Speed }\end{array}$ & Spot Size & $\begin{array}{c}\text { Laser Beam } \\
\text { Quality }\end{array}$ & $\begin{array}{c}\text { Cutting } \\
\text { Gas }\end{array}$ & Focal \\
Distance
\end{tabular}
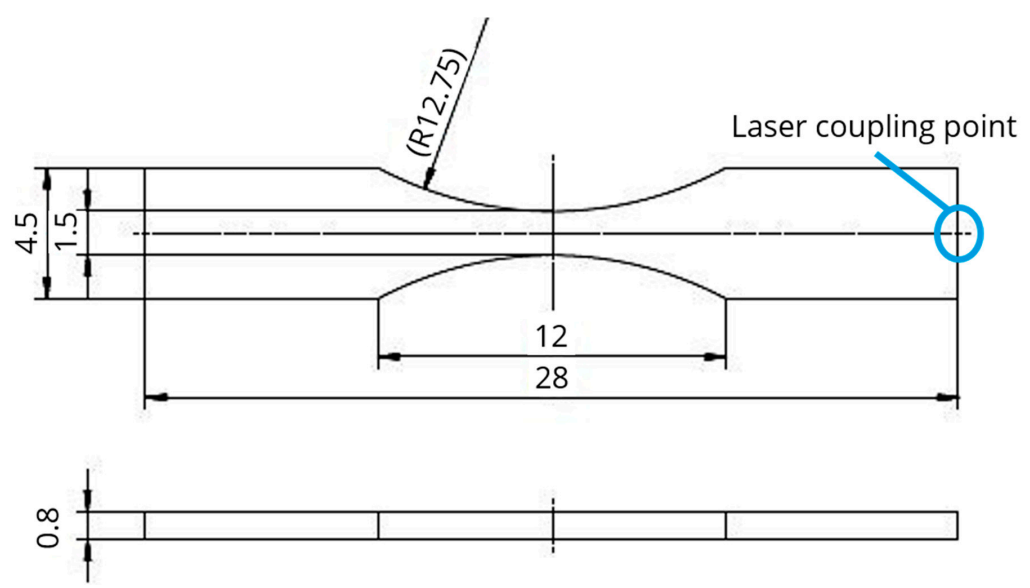

Figure 1. Fatigue sample geometry of Ti-6Al-4V-ELI with a marked laser coupling point. Dimensions in $\mathrm{mm}$.

The samples originally processed by laser-cutting were divided into three series for fatigue testing. Laser-cut samples (Ti-6Al_LC), barrel-grinded samples (Ti-6Al_BG), and mechanically polished samples (Ti-6Al_MP) were investigated in detail before fatigue testing to document and assess the surface quality and near surface microstructure (heat-affected zone-HAZ) after the laser-cutting process, as well as after the mechanical post-processing. Therefore, metallographic cross-sections were prepared and microstructure analysis was executed by means of light microscopy, as well as scanning electron microscopy (SEM-JEOL JSM 7800-JEOL Ltd., Tokyo, Japan). The surface relief and roughness after laser-cutting was evaluated by confocal microscopy (Leica DCM 3D - Leica Microsystems, Wetzlar, Germany) using the software Leica Map Premium 6.2.7487. The element analysis for microstructural mapping in the HAZ was realized by energy dispersive spectroscopy (EDS-Oxford Instruments, Abingdon, Great Britain), which was attached on the SEM column. The AZtecHKL software (version 3.0) was used for evaluation. 
All fatigue tests were carried out by a servo-electric load-frame (SEL 010 with software Loadframe SX 2.4-Fa. Thelkin, Winterthur, Switzerland) using an attached cooling device with pressurized air to hold the sample temperature during the fatigue tests at a constant room temperature level. The load-controlled tests were executed under tension-compression mode $(R=-1)$ with a frequency of $20 \mathrm{~Hz}$. The fatigue life limit $\mathrm{N}_{\mathrm{G}}$ was set to $2 \times 10^{6}$ cycles. To ensure homogeneous sine oscillations, several pre-tests were carried out to find the best parameter sets of the test system for the specific vibration behavior of the Ti-6Al-4V-ELI alloy. Subsequently, load-increase tests were executed to find the suitable load horizons. Finally, series testing was realized by means of the staircase method (15-18 samples per series).

The Ti-6Al_LC samples were tested with the original surface condition after laser-cutting. The melting drops were removed in the clamping range to ensure safe testing. The Ti-6Al_BG samples were additionally barrel grinded with standard titanium alloy parameter sets ( $4 \mathrm{~h}$ (rough grit) and $4 \mathrm{~h}$ (fine grit) at $1000 \mathrm{RPM}$, pyramidal polishing stones) in a vibratory grinding machine for small samples (Fa. Rösler, Untermerzbach, Germany). The Ti-6Al_MP samples were manually mechanically polished along the cyclic load direction stepwise from P400, P800; P1200 to a final grid size of P2500, which corresponds to an average roughness value of $\mathrm{Sa}<0.500 \mu \mathrm{m}$. The mechanical-polishing and barrel-grinding process were, thereby, carried out under constant fluid (water for mechanical polishing; grinding fluid for barrel (vibratory) grinding). Metallographic cross-sectioning documented the change in microstructure and HAZ as a consequence of the mechanical post-processing.

The fracture surface analysis was executed after the fatigue tests with SEM to locate crack initiation sites of all samples and to assess the possible connection between surface roughness-related notch effects due to laser-cutting and actual crack initiation after fatigue failure.

\section{Results and Discussion}

\subsection{Surface Quality}

The surface quality achieved due to the laser-cutting process with the particular cutting parameters applied (see Material and Methods section) is depicted in Figures 2 and 3. The Ti-6Al-4V-ELI alloy shows a distinctive surface relief with recognizable additional melting drops on the lower cutting edge. On the upper cutting edge, an irregular distributed terrace-like surface structure can also be identified (Figure 3a). Both observed features can be traced back to the influence of the laser-cutting parameters applied. The melting drops are caused by an insufficient pressure of the inert argon gas atmosphere. Whereas on the upper cutting edge, the occurring melting drops could be sufficiently removed, the lower cutting edge is less accessible, demanding a higher pressurized air stream to successfully remove all residual melting drops. As a consequence, the necessary argon pressure has to be well over 6 bar to homogeneously remove melting drops on the whole cutting edge.

The irregular terrace-like structure can be assigned to an influence throughout the interaction of the laser and Ti-6Al-4V-ELI but not directly explained till now. Further evaluation is, therefore, necessary. However, optimized laser-cutting parameters will improve the surface quality to a great extent, and therefore, also has a positive effect on the resulting fatigue strength of the Ti-6Al-4V-ELI alloy. The average height parameters of the laser-cut surface relief are measured according to ISO 25178 by means of confocal microscopy. Several $\mu \mathrm{m}$ in distance between the relief hills and valleys (Figure 2a) are identified, which concludes a significant surface roughness. Since fatigue failure in the LCF $\left(<1 \times 10^{3}-10^{4}\right.$ cycles) and HCF (up to $1 \times 10^{7}$ cycles) regime is mostly triggered by the stress concentration at distinct surface flaws leading to early crack initiation and propagation, pronounced surface roughness is detrimental for the expected fatigue strength and endurable cycles [10-12]. The Ti-6Al_LC samples with a surface quality as depicted in Figure 2 could, therefore, be expected to cause a dominant concentration of cyclic stress in the relief valleys during fatigue. In addition, Ti-6Al-4V is known for its high notch sensitivity, which was the subject of detailed studies in the past $[3,10,14,19]$, demonstrating a strong decrease of fatigue strength correlating with higher surface roughness and 
resulting notch factors. Comparing the surface quality after laser-cutting (Ti-6Al_LC) with the sample series of Ti-6Al-BG and Ti-6Al_MP, the latter ones show a significantly improved surface quality, which correlates also with lower surface roughness. The mechanically polished surfaces of the Ti-6Al_MP samples are completely free of visible micro-notches which is a result of the homogeneous polishing to a grit size of P2500 and corresponds to a surface quality of $S_{a}<0.500 \mu \mathrm{m}$. In comparison to the as-cut surface, as well as the barrel grinded surface, this post-processing represents the best surface quality for the fatigue samples in the study presented.

(a)

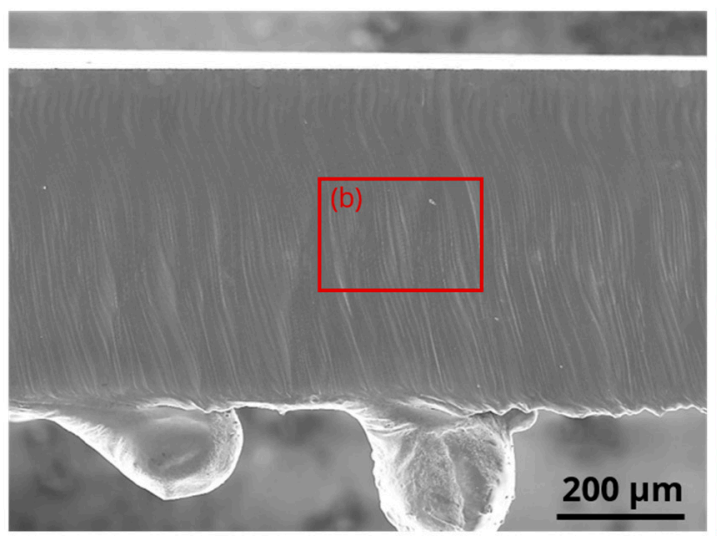

(b)

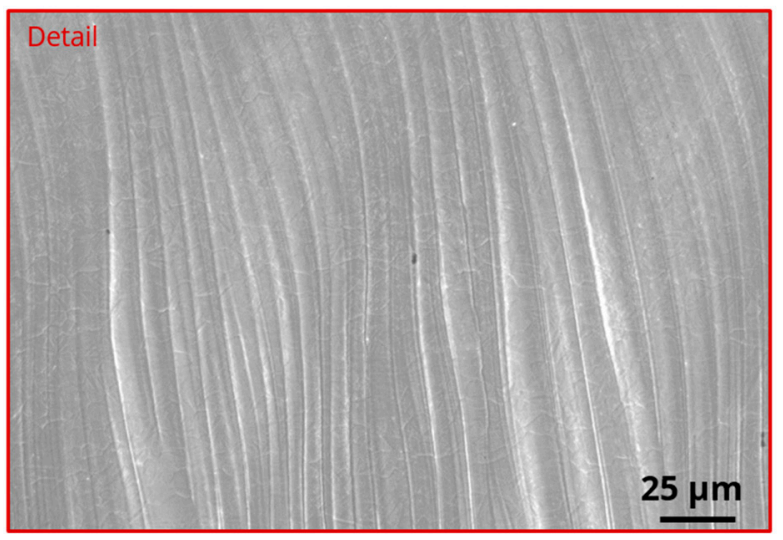

Figure 2. Obtained quality of the laser-cut surfaces of Ti-6Al-4V-ELI with non-optimized laser cutting conditions: (a) Overview SEM-image of the cutting edge with residual melting drops at the lower part; (b) Detailed SEM-image showing a wavy, irregular surface structure after the laser-cutting process.

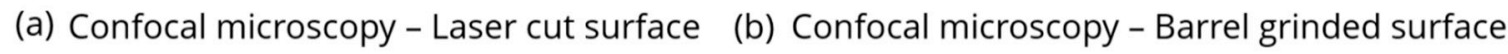

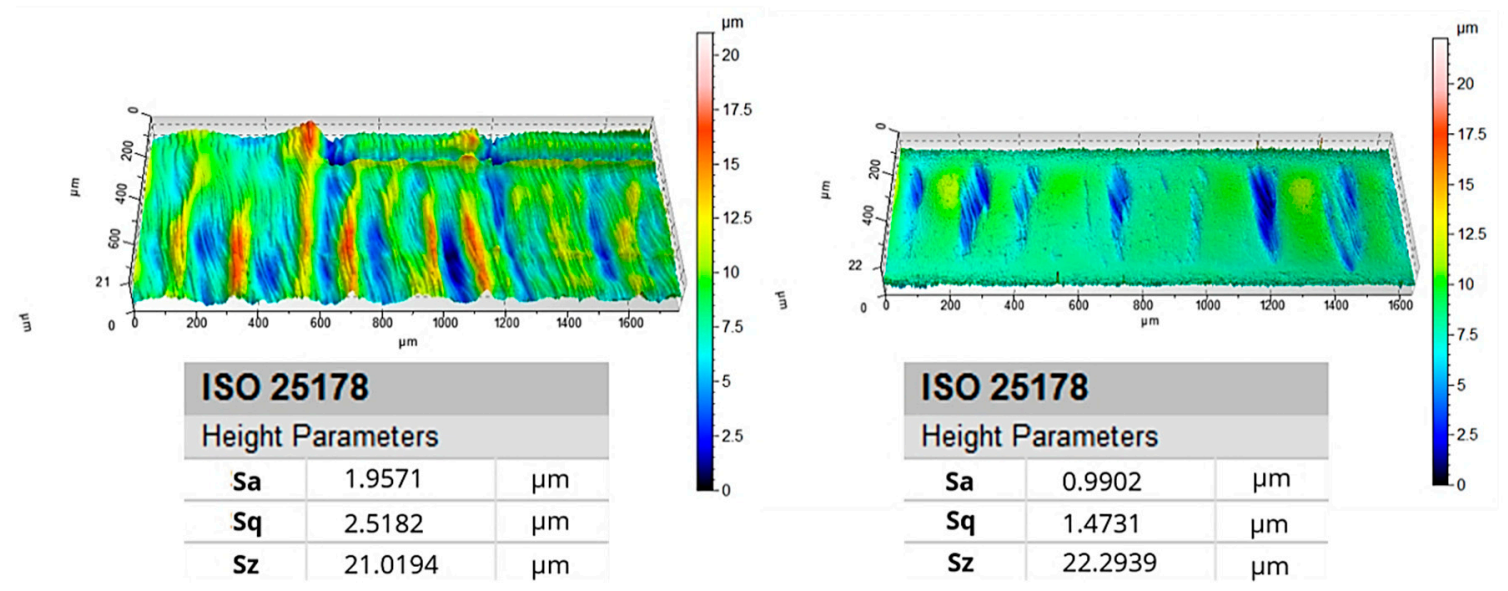

Figure 3. Typical representative surface reliefs by means of confocal microscopy (normalized) of Ti-6Al-4V-ELI with average height parameters: (a) Surface after laser-cutting; (b) Surface after barrel-grinding-Improvement of surface quality.

Barrel-grinded surfaces, on the other hand, show a strong improvement in surface quality and less roughness, but retained micro-notches are visible in the center area of the cutting edges (Figure 3b). These micro-notches could act as possible crack initiation sites in the application, which was already shown in detail in one of the authors previous studies of laser-cut $\beta$-titanium, as well as $\alpha$-titanium for fatigue samples and osteosynthesis plates [12]. This leads to the conclusion that the barrel-grinding process is an effective tool to reduce detrimental laser-cutting effects, but only under the prerequisite of already optimized laser-cutting parameters for a specific material. Without the improvement of the original laser-cut surface quality, micro-notches seem to remain in the center area of the laser-cutting edges playing a decisive role for achievable fatigue strength in the application. 


\subsection{Microstructural Development of the Heat-Affected Zone (HAZ)}

The microstructural response to the thermal input from processing in the heat-affected zone is alongside the surface quality, the most important factor to define the influence of the laser-cutting process regarding the fatigue behavior. Possible microstructural changes compared to the initial $\alpha+\beta$-microstructure could be expected for Ti-6Al-4V-ELI since the laser-cutting temperature lies well above the $\beta$-transus temperature of this alloy $\left(\approx 980^{\circ} \mathrm{C}[35]\right)$. Hence, the laser-cutting process could change the surface-near microstructure caused by the local heating and subsequent self-quenching.

The HAZ in a cross-sectional view at the upper cutting edge depicted in Figure 4 clearly demonstrates the change in microstructure from equiaxial $\alpha+\beta$ into acicular martensitic $\alpha^{\prime}$ with retained $\beta$, the latter being proven by the EDS-analysis showing a higher V-content as a strong $\beta$-phase stabilizer. The transformation into martensitic $\alpha^{\prime}$ is, thereby, associated with the self-quenching process, which is fast enough to transform the initial equiaxial $\alpha$-phase (5-10 $\mu \mathrm{m}$ in grain size) to fine acicular $\alpha^{\prime}$ martensite. The retained $\beta$-phase can be explained by the very fast processing time and holding over $\beta$-transus not allowing the $\beta$-phase also to transform into $\alpha^{\prime}$ martensite. The lower cutting edge with residual melting drops (Figure 2a) shows, by contrast, two distinctive zones (Figure 5-Cross-sectional view). A small surface layer (average thickness $10-15 \mu \mathrm{m}$ ) consists solely of acicular $\alpha^{\prime}$ without $\beta$-phase. The second zone, which develops with growing distance to the free surface, consists of $\alpha^{\prime}$ and $\beta$-phase, comparable to the upper cutting edge, followed by the initial $(\alpha+\beta)$-microstructure. The development of the different microstructural zones could be explained with the self-quenching gradient from the laser-cutting temperature, which causes different quenching speeds towards the sample interior and, therefore, possible zone formation. However, in order to explain the pronounced microstructural difference of upper and lower cutting edges, more detailed information of the temperature distribution related to the process parameters would be necessary.

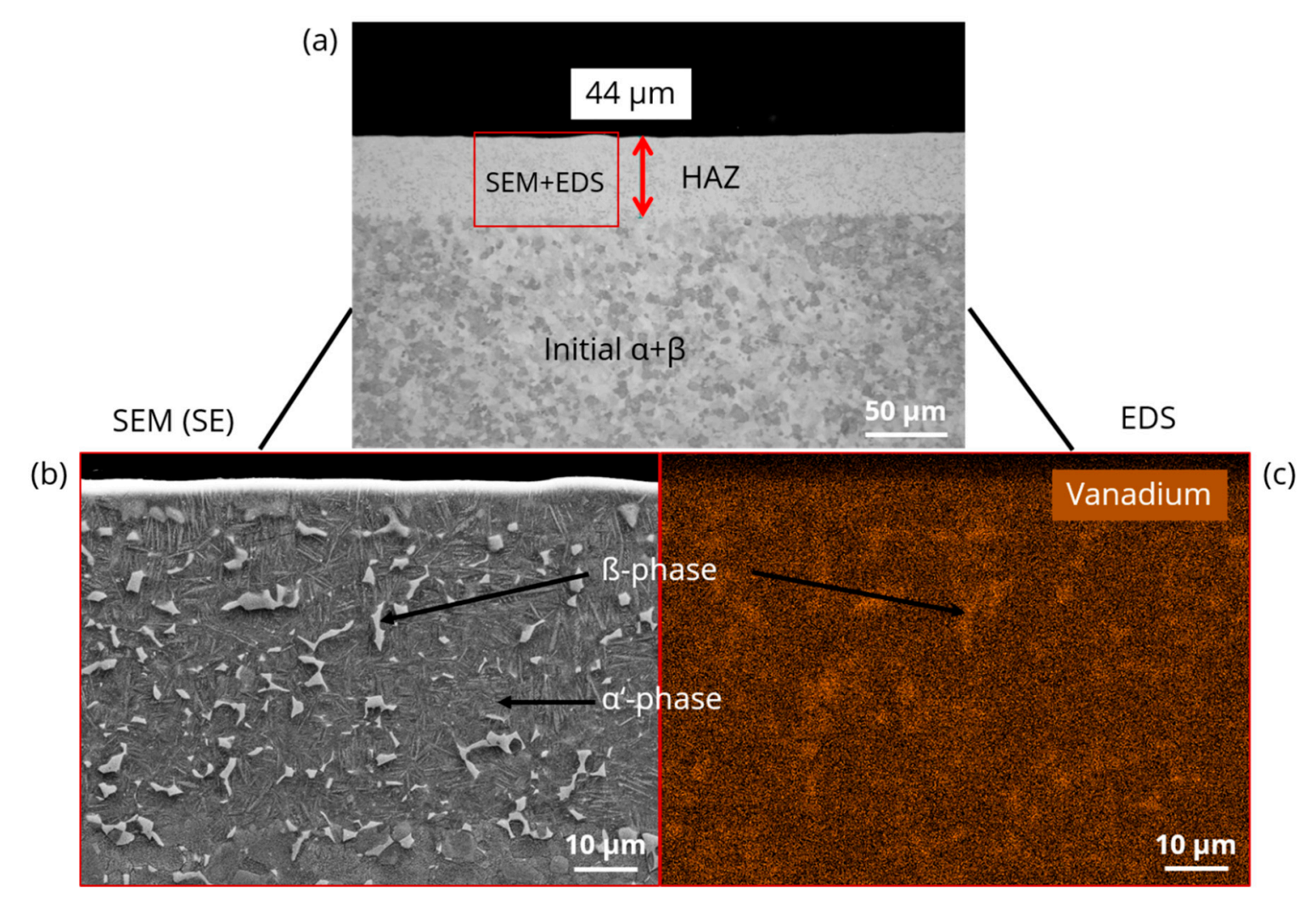

Figure 4. Typical HAZ at the upper cutting edge developed during the laser cutting process (Ti-6Al_LC sample): (a) Light microscopic image representing an overview; (b) SEM-image (SE cross-section) of the HAZ microstructure with $\alpha^{\prime}$-martensite; (c) Element analysis (EDS) showing the higher vanadium content ( $\beta$-stabilizer) in the $\beta$-phase of the HAZ. 


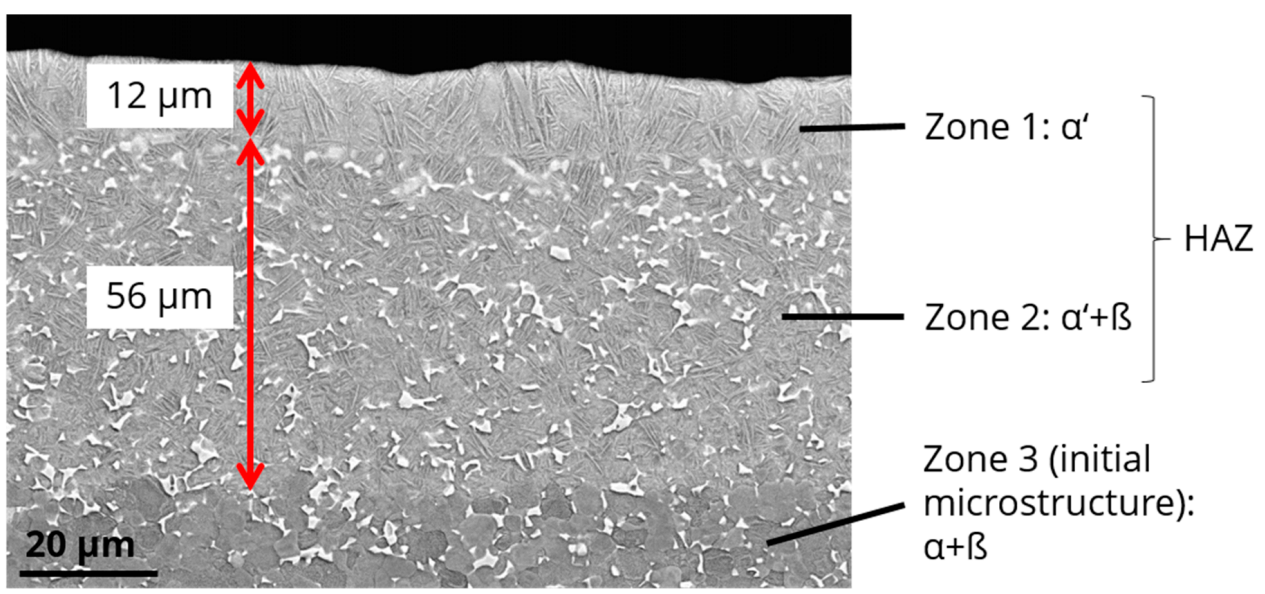

Figure 5. SEM image (BSE cross-section) of the HAZ at the lower cutting edge of a Ti-6Al_LC sample showing three distinctive microstructural zones after laser-cutting consisting of martensitic $\alpha^{\prime}$-phase and retained $\beta$-phase.

The measured thickness of the HAZ $(\approx 40-70 \mu \mathrm{m})$ depends on the occurrence of the different zones and is more pronounced at the lower cutting edge with the additional $\alpha^{\prime}$-zone. The optimized laser-cutting parameters, such as speed and power would contribute to a significant less pronounced HAZ. Furthermore, the application of a pulsed laser is expected to have a positive effect on the local microstructural changes due to the very short processing window and heating over the $\beta$-transus temperature [36].

With the mechanical post-processing of the laser-cut surface, not only the surface quality is improved, as was discussed in Section 3.1, but also the HAZ is significantly affected. The result is depicted in Figure 6 showing a significant reduction of the HAZ thickness. The barrel-grinding process creates, thereby, a decrease of about $50 \%$ HAZ compared to the initially observed HAZ without any mechanical post-processing. The reduction is independent of upper or lower cutting edge but shows irregularities at the crossover region of the radius and clamping range. This phenomenon can be explained by the mechanism of the barrel grinding process itself, which is based on a rotary or vibratory grinding process between the sample and a specific grinding stone, as well as an abrasive medium [37,38]. The careful selection and adaption of this process is a prerequisite for an optimal and homogeneous grinding result. The complex geometries and very poor initial surface quality are prerequisites that may have an adverse effect on the grinding quality. Insufficient grinding results negatively influence the fatigue properties with residual micro-notches on geometrically inaccessible locations on samples or components [12].

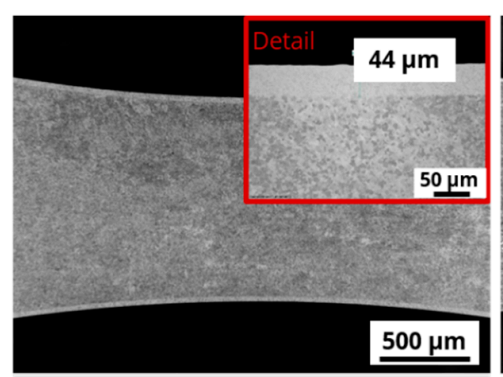

Laser-cut (as-cut initial condition)

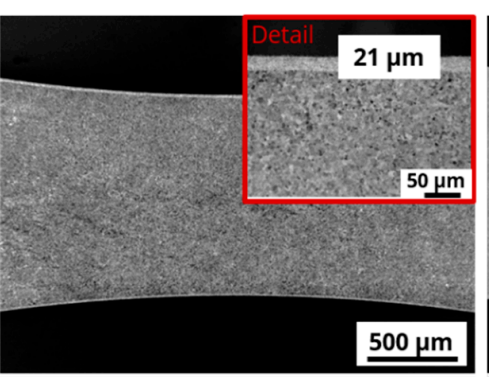

Barrel grinding

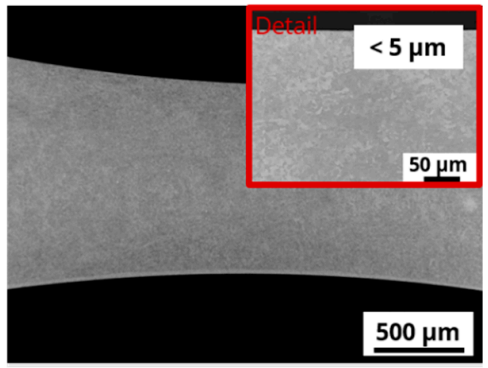

Mechanical polishing

\section{HAZ-thickness due to mechanical post-processing}

Figure 6. Thickness of the HAZ dependent on the mechanical post-processing showing a significant decrease in measured HAZ-thickness at the upper cutting edge. 
The results of mechanical polishing are also depicted in Figure 6. An almost complete removal of the HAZ is observed. However, it has to be pointed out that in the study presented, the mechanical polishing was executed hand-made, leading to slight variations regarding the extent of removal of the HAZ for the overall number of samples.

\subsection{Fatigue Results and Crack Initiation}

The fatigue test results for all the three sample series are shown in Figure 7a. Remarkable differences are recognizable between the samples with the as-cut surface (Ti-6Al_LC) and the sample series with additional post-processing of the surface (Ti-6Al_BG and Ti-6Al_MP). The results for the as-cut samples show a significant scatter and the lowest fatigue strength $\left(\sigma_{a D, 50 \%}=235 \mathrm{MPa}\right)$ for a fatigue life limit of $N_{G}=2 \times 10^{6}$. A superior fatigue strength was reached for the series with barrel-grinded surface (351 MPa) and mechanically polished surface (363 MPa). These values represent an increase of around 50\% compared to the original laser-cut surface condition. However, while comparing the fatigue strengths obtained for the different sample conditions, it has to be considered that the as-cut condition represents a more or less "worst-case" condition since the process parameters were chosen on the basis of practical knowledge and were not yet optimized. The optimized laser-cutting parameters result in strongly decreased surface roughness and improved fatigue strength. The scattering of the results is less distinctive with mechanical post-processing of the surface and can be attributed to a higher surface quality with less surface roughness, and therefore, less possible notches acting as stress concentrators during fatigue loading. For the barrel grinded surface condition; however, micro-notches remain in the center area of the laser-cut edges, which was exemplarily shown in Figure 3. In comparison to the process of mechanical polishing, which displays the best overall fatigue behavior, barrel grinding of the Ti-6Al-4V-ELI alloy must be optimized, especially in case of homogeneity and removal of all surface notches.

(a)

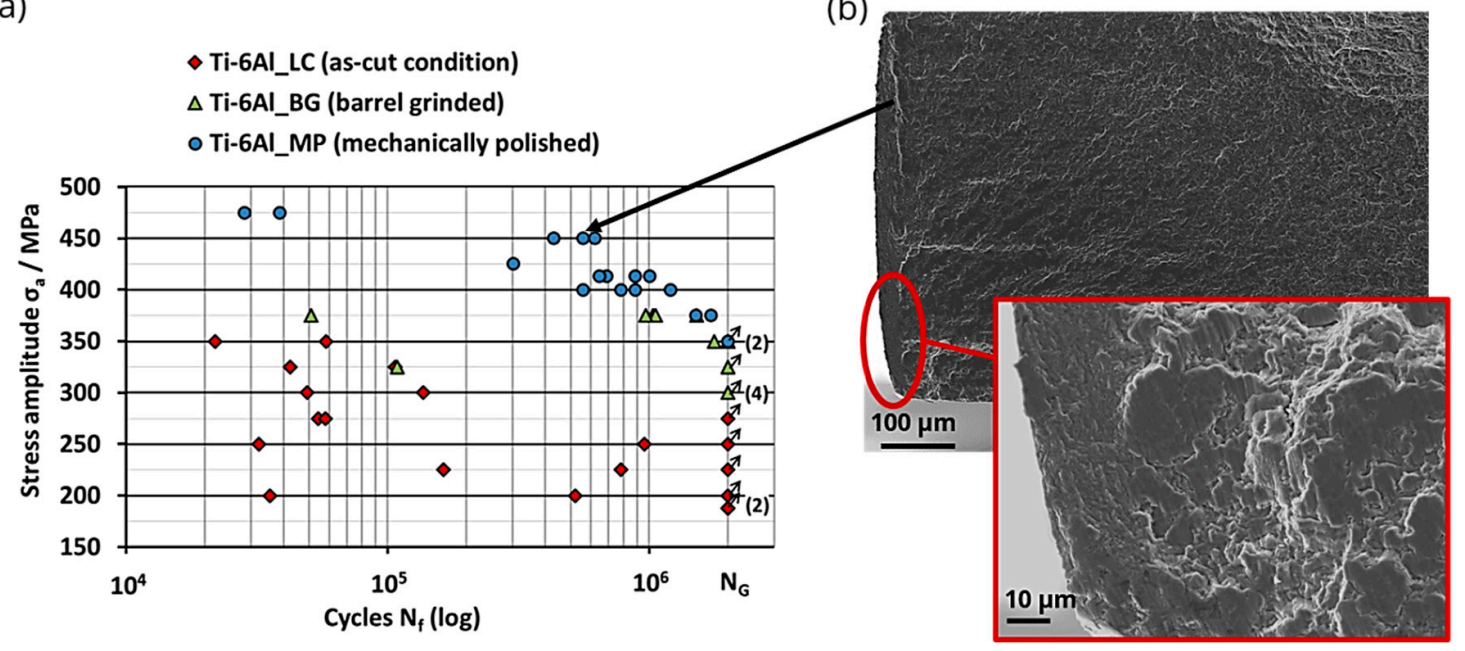

Figure 7. Fatigue results of the Ti-6Al-4V-ELI alloy: (a) S-N diagram for all three tested sample series; (b) Fracture surface analysis for a Ti-6Al_MP sample with mechanically polished surface conditions including the crack initiation site on the laser-cut edge directly near the sample corner after fatigue failure at $450 \mathrm{MPa}$ and $6.2 \times 10^{5}$ cycles.

Fatigue crack initiation sites for all Ti-6Al-4V-ELI samples are located at the sample edges and corners. There are distinct differences between the three tested series, which are depicted in Figures $7 \mathrm{~b}$, 8 and 9. For the mechanically polished surface condition (Figure $7 \mathrm{~b}$ ), fatigue cracks initiate at the laser-cut edges or the flat sample edges, but primarily at or directly near the sample corners. The reason for this behavior is the overall good surface quality and comparable surface roughness of all sample edges with no distinctive micro-notches. In this case, the sample corners act normally as the 
highest stress concentrator under fatigue loading for flat sample geometries [39]. The consequence is a preferred fatigue crack initiation at these sites for the Ti-6Al_MP samples.

(a) Side view on laser-cut edge

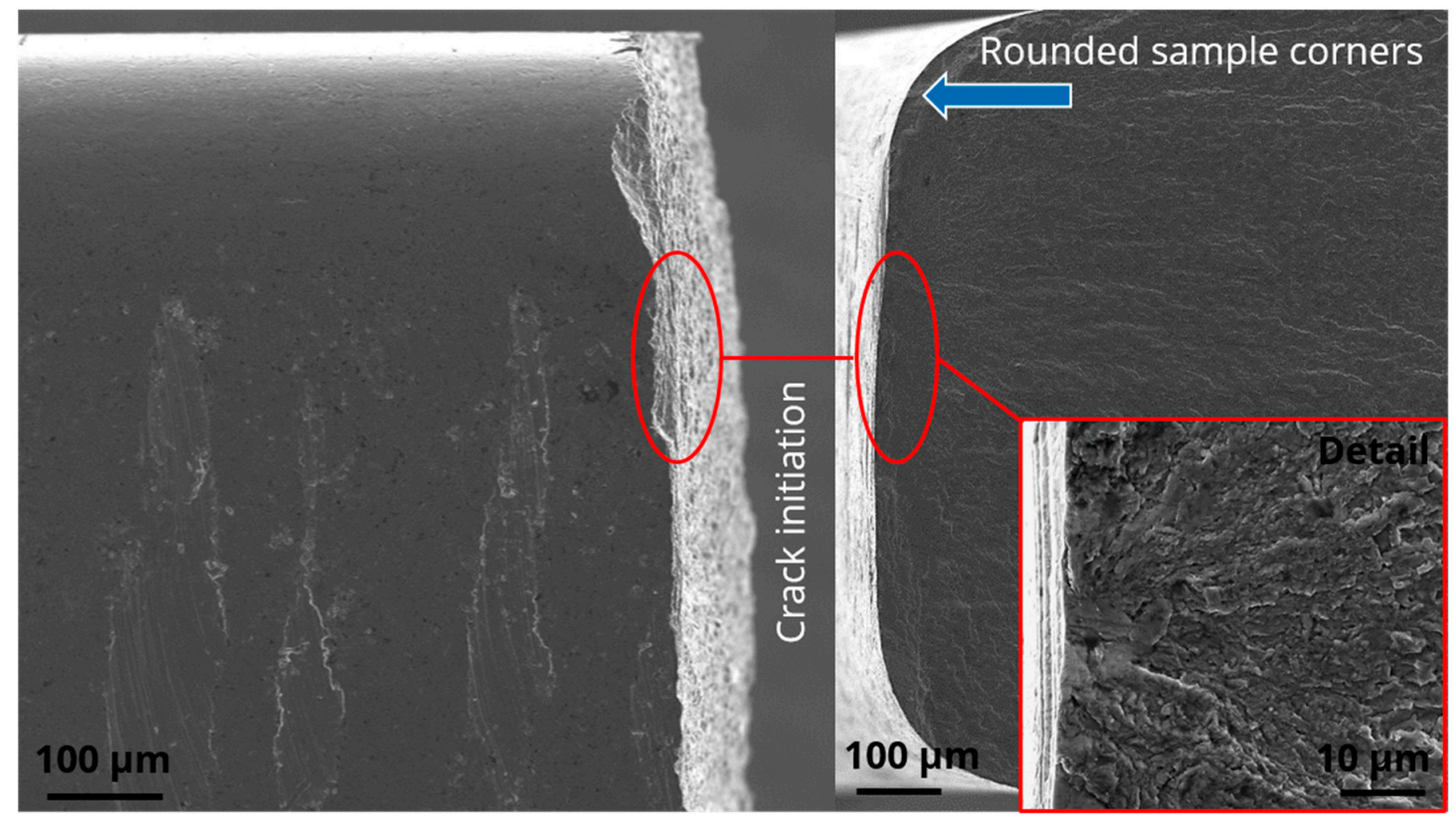

Figure 8. Fractography of a Ti-6Al_BG sample with barrel-grinded surface condition failed at $375 \mathrm{MPa}$ and $5.1 \times 10^{4}$ cycles: (a) SEM (SE) analysis of the laser-cut edge with detectable notches; (b) The corresponding fracture surface with detailed view on fatigue crack initiation.

(a)

(b)

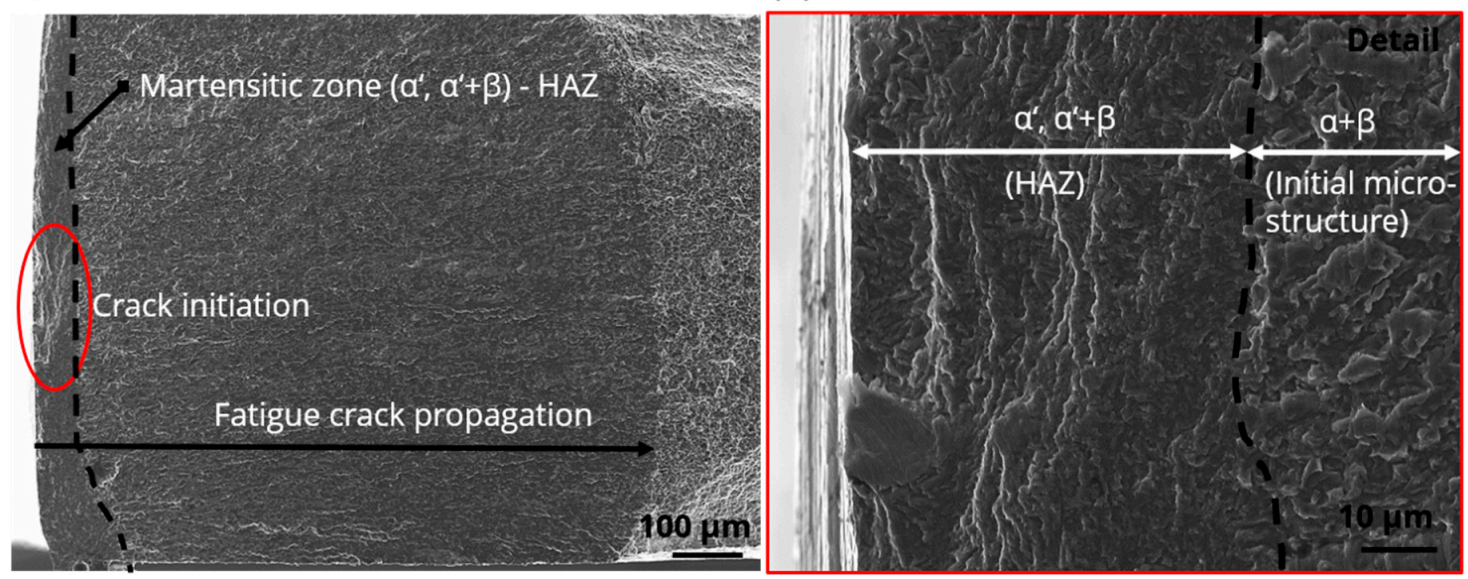

Figure 9. Fractography of a Ti-6Al_LC sample with as-cut surface condition failed at $200 \mathrm{MPa}$ and $5.2 \times 10^{5}$ cycles: (a) SEM (SE) analysis of the fractured surface with identification of the martensitic zone; (b) The corresponding detailed view at the fatigue crack initiation point.

The sample series with the barrel-grinded surface condition (Ti-6Al_BG) showed, on the contrary, fatigue crack initiation always on the laser-cut edges, which is exemplarily depicted in Figure 8. Furthermore, the crack initiation sites are not always located at the smallest loaded cross-section but rather in the radius section. The cause for this behavior can be assigned to the remaining and detected surface notches after the barrel-grinding process in the middle of the laser-cut edges (Figures 3 and 8a). The irregular size and distribution of these remaining notches leads consequently to stress concentration, crack initiation, and failure at the biggest notches, and these can be located outside the smallest loaded cross-section. Associated with the effective lower overall surface roughness of 
the laser-cut edges and the observed, particularly rounded, sample corners (Figure 8b-lower stress concentration sites), all Ti-6Al_BG samples are expected to fail at one of the remaining notches.

The sample series with the initial as-cut surface condition (Ti-6Al_LC) reveal almost similar fatigue crack initiation points as the Ti-6Al_BG samples. Due to the significantly stronger local surface roughness (compared to the flat sample edges) caused by the laser-cutting for the process parameters applied, fatigue failure could always be directly assigned to the crack initiation at the laser-cut edges, which is depicted in Figure 9 in the fracture surface analysis. Pronounced irregularity of the surface profile leads subsequently to the high scattering of the fatigue results and the decreased fatigue strength for the Ti-6Al_LC sample series. At this point, it should be pointed out that, irrespective of the severe geometrical irregularity of the dross formation (see Figure 2a) due to a lack of process optimization, fatigue crack initiation always started from an extrusion/intrusion from the surface relief and not from a dross. The optimized cutting conditions improve this behavior and lead to significantly improved fatigue strength, although the formation of a slight surface relief cannot be completely avoided and may have an effect on the fatigue behavior. By comparing the fatigue behavior of the barrel-grinded and the as-cut condition, it becomes obvious that even marginal remains of the surface relief can result in early failure with values for the barrel grinded condition close to the upper scattering range of the as-cut results. The fatigue results obtained for the stress amplitudes between $300 \mathrm{MPa}$ and $350 \mathrm{MPa}$ demonstrate the particularly high sensitivity of the fatigue behavior of Ti-6Al-4V-ELI on surface flaws with early failures $\left(N<2 \times 10^{5}\right)$, as well as run-out samples $\left(N=2 \times 10^{6}\right)$ for the same stress level.

An aspect to be considered for all three sample series, but especially for the as-cut surface condition, is the presence of the HAZ caused by the laser-cutting. The martensitic zone $\left(\alpha^{\prime}\right.$ and $\alpha^{\prime}+\beta-$ Figures 4 and 5) is especially pronounced in the as-cut surface condition (Figure 6) and expected to influence the fatigue behavior, although an optimized laser-cutting process will significantly decrease HAZ development. Due to a higher strength and more interphase boundaries, this phase can have a positive effect on the fatigue strength or the fatigue crack growth behavior of Ti-6Al-4V [3,4,29]. However, in connection with higher or irregular surface roughness, $\alpha^{\prime}$-martensite can have an opposing effect and decrease the fatigue strength due to higher sensitivity for crack initiation and growth, which can be explained with higher hardness and lower ductility of $\alpha^{\prime}$ compared with the initial $\alpha+\beta$ microstructure $[16,19]$. In the study presented, a pronounced HAZ can clearly be observed for all samples with an as-cut surface condition (Figure 9). The specific implication on the resulting fatigue strength seems; however, not significant due to the superior effect of the surface roughness, which is supported by the fact of highly scattered results for Ti-6Al_LC samples. In order to clearly identify an influence of the HAZ on the early stages of fatigue crack initiation and growth, further investigations with pre-notched samples would be necessary in order to quantitatively measure early crack growth rates in the HAZ. For the sample series with mechanical post-processing, HAZ was also observed, but in a less distinctive manner. The superior role of surface roughness is however confirmed with crack initiation on specific notches for the barrel grinded surface condition or near sample corners for the mechanically polished surface condition. It should be mentioned that possible residual stresses introduced in the near-surface area and their potential influence on the fatigue strength has not been part of the investigation of this study. However, this effect must be taken into consideration when further elucidating the superior fatigue behavior of the mechanically polished condition.

A comparison of the findings in the study presented with fatigue properties in the literature show a good correlation with the lower range of possible fatigue strengths for the $(\alpha+\beta)$ Ti-6Al-4V alloy $[14,15,25,26,29]$. The overall fatigue properties of Ti-6Al-4V are, thereby, dependent on various influencial factors regarding the microstructure, the fatigue test conditions, as well as the fatigue geometry, composition and additional surface treatments. Furthermore, the role of thermal and residual stresses and their influence on the fatigue behavior must be taken into account $[2-4,16,29]$. Although the residual stress measurements were not executed in the study presented, it can be assumed that compressive residual stresses are introduced during the process of mechanical finishing. For the as-cut condition, the higher hardness of the $\alpha^{\prime}$-zone indicates an increase in mechanical strength; however, 
regarding the low surface quality of the as-cut condition, this general increase in strength can be assumed to be more susceptible for early fatigue failure and crack initiation due to an increased notch sensitivity. The compressive residual stresses introduced by means of mechanical polishing, on the other hand, contribute to an enhanced fatigue strength, and combined with an improved surface quality will result in an improved fatigue behavior [3,4,29]. The discrepancies in fatigue strength of Ti-6Al-4V_ELI in the study presented clearly correlates with the surface quality of each processing condition and, in particular, the resulting significant notch effects caused by the non-optimized laser-cutting process. In consequence, an optimization of the used laser-cutting parameters could be expected to significantly improve fatigue behavior.

The results of the as-cut surface condition impressively demonstrate the detrimental effect of the surface roughness with exceptional low fatigue strength $\left(\sigma_{a D, 50 \%}=235 \mathrm{MPa}\right)$ and pronounced scatter of the fatigue results. However, optimum laser-cutting parameters decrease this negative effect and strongly enhance fatigue behavior, because of the direct correlation of laser-cutting parameters and resulting surface quality. The barrel-grinding process (Ti-6Al_BG samples) results in a strong increase in fatigue strength, but is, nevertheless, determined by the dominating influence of the remaining notch profile in the center area of the laser-cut edges. The difference in fatigue strength compared to the mechanically polished surface condition (Ti-6Al_MP samples_Figure 7) can mainly be explained by the effect of the flat sample geometry and the consequential influence of the sample corners. These corners act as preferential, and possible early crack initiation sites in case of the Ti-6Al_MP samples with mechanically polished surface, whereas for Ti-6Al_BG samples with barrel grinded surface condition observed significant rounding of the corners (Figure 8) has the opposite effect. Hence, both sample series with mechanical post-processing of the surface exhibit almost similar fatigue behavior. The influence of the underlying microstructure and investigated HAZ seems to play only a circumstantial role compared to the dominant influence of the surface roughness. In consequence, the optimization of the surface roughness has to be the essential goal for laser cutting of Ti-6Al-4V-ELI in order to avoid early fatigue failure in application. Mechanical post-processing by means of barrel (vibratory) grinding or polishing seems to be an excellent choice for achieving this goal due to clear enhancement of fatigue strength caused by the minimization of arising surface roughness during laser cutting. Furthermore, residual compressive stresses could be introduced, positively influencing stress concentration and subsequent crack initiation during fatigue loading. All these factors are controlled by the grinding and polishing parameters, as well as the exact alloy composition, heat treatment condition, and original surface quality due to laser cutting, which, if mutually adjusted, hold the potential for significant fatigue life improvement. The mechanical post-processing, such as barrel grinding, can easily be implemented in industrial process chains and quality control. The downside is, on the other hand, the control of dimensions, especially in the case of maintaining the original edge proportion, which are prone to rounding (Figure $8 \mathbf{b}$ ). Only recognition of all influencing effects can, therefore, lead to economic and positive post-processing with subsequent utilization of the full material potential under fatigue loading.

\section{Conclusions}

The study presented investigated the fatigue behavior of the medical implant alloy Ti-6Al-4V-ELI processed by laser-cutting and the consequences of additional surface post-processing on the fatigue properties with regard to surface roughness and HAZ. The fatigue behavior of the as-cut surface condition was compared with mechanically polished surfaces, as well as with barrel-grinded surfaces. The results can be summarized and concluded as follows:

- The surface relief introduced by the non-optimized laser-cutting influences the fatigue behavior of Ti-6Al-4V-ELI significantly. For the process parameters featured in this study, fatigue strength of the as-cut condition results in a drastic decrease of the fatigue strength compared to the additionally surface-treated condition. However, the difference in fatigue strength observed in this study will be controllable by optimizing the laser-cutting parameters. 
- The main reason for the superior fatigue strength of the post-processed conditions is a minimized surface roughness, which in turn is responsible for higher resistance against fatigue crack initiation on macro- and micro-notches originally caused by the laser-cutting.

- The process of barrel-grinding after the laser-cutting was effective but revealed retained surface roughness in the center area of the cutting edges, which acts as preferred crack initiation sites compared to the particularly rounded sample corners.

- Mechanically polished samples always failed at or near the sample corners, which is caused by a stress concentration on these sites.

- The HAZ consisting of martensitic $\alpha^{\prime}$ and $\beta$ along distinctive surface and subsurface zones was analyzed and does not play a significant role in early fatigue failure, which instead was dominated by the surface roughness. Nevertheless, the applied mechanical post-processing led to an almost complete removal of the HAZ.

- To avoid early fatigue failure in the application, an optimization of the laser-cutting parameters is crucial in order to obtain better surface quality. This allows the required post-processing to improve the surface roughness further and, therefore, the fatigue strength. However, both processes, laser-cutting and mechanical post-processing, have to be optimized in the dependence of the specific alloy composition and fatigue behavior of Ti-6Al-4V-ELI.

Author Contributions: Conceptualization-A.R., M.Z.; Methodology-A.R.; Investigation-A.R., A.T.Z.; Data analysis/curation-A.R., A.T.Z.; Writing—original draft preparation-A.R.; Writing-review and editing-M.Z., A.R.; Project administration-A.R., M.Z.; Funding acquisition-M.Z.

Funding: This research was funded by Deutsche Forschungsgemeinschaft (DFG) under the grant number 402380578 (ZI 1006/14-1).

Acknowledgments: The authors thank Nikolai Schröder and the working group laser cutting at IWS for carrying out the laser cutting as well as Robert Kühne and Sebastian Schettler from the department Materials Characterization and Testing for helpful thematic discussions regarding the fatigue testing. Further thanks to Clemens Grahl for confocal microscopy as well as the metallographic team of the IWS, Lars Ewenz and Sebastian Schöne for sample preparation and assistance.

Conflicts of Interest: The authors declare no conflict of interest.

\section{References}

1. Banerjee, D.; Williams, J.C. Perspectives on titanium science and technology. Acta Mater. 2013, 61, 844-879. [CrossRef]

2. Leyens, C.; Peters, M. Titanium and Titanium Alloys. Fundamentals and Applications, 1st ed.; Wiley-VCH: Weinheim, Germany, 2003.

3. Lütjering, G.; Williams, J.C. Titanium, 2nd ed.; Springer: Berlin, Germany, 2007.

4. Bache, M. Processing titanium alloys for optimum fatigue performance. Int. J. Fatigue 1999, 21, $105-111$. [CrossRef]

5. Chandravanshi, V.; Prasad, K.; Singh, V.; Bhattacharjee, A.; Kumar, V. Effects of $\alpha+\beta$ phase deformation on microstructure, fatigue and dwell fatigue behavior of a near alpha titanium alloy. Int. J. Fatigue 2016, 91, 100-109. [CrossRef]

6. Ezugwu, E.O.; Wang, Z.M. Titanium alloys and their machinability-A review. J. Mater. Process. Technol. 1997, 68, 262-274. [CrossRef]

7. Steen, W.M. Laser Material Processing, 3rd ed.; Springer: London, UK, 2003.

8. Davim, J.P. Lasers in Manufacturing; Wiley-VCH: Chichester, UK, 2013.

9. Yilbas, B.S. The Laser Cutting Process. Analysis and Applications; Elsevier Science: San Diego, CA, USA, 2017.

10. da Silva, P.S.C.P.; Campanelli, L.C.; Escobar Claros, C.A.; Ferreira, T.; Oliveira, D.P.; Bolfarini, C. Prediction of the surface finishing roughness effect on the fatigue resistance of Ti-6Al-4V ELI for implants applications. Int. J. Fatigue 2017, 103, 258-263. [CrossRef]

11. Pessoa, D.F.; Herwig, P.; Wetzig, A.; Zimmermann, M. Influence of surface condition due to laser beam cutting on the fatigue behavior of metastable austenitic stainless steel AISI 304. Eng. Fract. Mech. 2017, 185, 227-240. [CrossRef] 
12. Reck, A.; Pilz, S.; Calin, M.; Gebert, A.; Zimmermann, M. Fatigue properties of a new generation $ß$-type $\mathrm{Ti}-\mathrm{Nb}$ alloy for osteosynthesis with an industrial standard surface condition. Int. J. Fatigue 2017, 103, 147-156. [CrossRef]

13. Yang, J.; Sun, S.; Brandt, M.; Yan, W. Experimental investigation and 3D finite element prediction of the heat affected zone during laser assisted machining of Ti6Al4V alloy. J. Mater. Process. Technol. 2010, 210, 2215-2222. [CrossRef]

14. Eylon, D.; Pierce, C.M. Effect of microstructure on notch fatigue properties of Ti-6Al-4V. Metall. Trans. A 1976, 7, 111-121. [CrossRef]

15. Stráský, J.; Janeček, M.; Harcuba, P.; Bukovina, M.; Wagner, L. The effect of microstructure on fatigue performance of Ti-6Al-4V alloy after EDM surface treatment for application in orthopaedics. J. Mech. Behav. Biomed. Mater. 2011, 4, 1955-1962. [CrossRef]

16. Sonntag, R.; Reinders, J.; Gibmeier, J.; Kretzer, J.P. Fatigue performance of medical Ti6Al4V alloy after mechanical surface treatments. PLoS ONE 2015, 10, e0121963. [CrossRef] [PubMed]

17. Nalla, R.K.; Altenberger, I.; Noster, U.; Liu, G.Y.; Scholtes, B.; Ritchie, R.O. On the influence of mechanical surface treatments—-deep rolling and laser shock peening—on the fatigue behavior of Ti-6Al-4V at ambient and elevated temperatures. Mater. Sci. Eng. A 2003, 355, 216-230. [CrossRef]

18. Nie, X.; He, W.; Zhou, L.; Li, Q.; Wang, X. Experiment investigation of laser shock peening on TC6 titanium alloy to improve high cycle fatigue performance. Mater. Sci. Eng. A 2014, 594, 161-167. [CrossRef]

19. Guilherme, A.S.; Henriques, G.E.P.; Zavanelli, R.A.; Mesquita, M.F. Surface roughness and fatigue performance of commercially pure titanium and Ti-6Al-4V alloy after different polishing protocols. J. Prosthet. Dent. 2005, 93, 378-385. [CrossRef]

20. Arif, A.F.M.; Yilbas, B.S. Thermal stress developed during the laser cutting process: Consideration of different materials. Int. J. Adv. Manuf. Technol. 2008, 37, 698-704. [CrossRef]

21. Sharma, A.; Yadava, V. Experimental analysis of Nd-YAG laser cutting of sheet materials-A review. Opt. Laser Technol. 2018, 98, 264-280. [CrossRef]

22. Sheng, P.S.; Joshi, V.S. Analysis of heat-affected zone formation for laser cutting of stainless steel. J. Mater. Process. Technol. 1995, 53, 879-892. [CrossRef]

23. Tamilarasan, A.; Rajamani, D. Multi-response optimization of Nd:YAG laser cutting parameters of Ti-6Al-4V superalloy sheet. J. Mech. Sci. Technol. 2017, 31, 813-821. [CrossRef]

24. Pandey, A.K.; Dubey, A.K. Modeling and optimization of kerf taper and surface roughness in laser cutting of titanium alloy sheet. J. Mech. Sci. Technol. 2013, 27, 2115-2124. [CrossRef]

25. Mower, T.M. Degradation of titanium $6 \mathrm{Al}-4 \mathrm{~V}$ fatigue strength due to electrical discharge machining. Int. J. Fatigue 2014, 64, 84-96. [CrossRef]

26. Carrion, P.E.; Shamsaei, N.; Daniewicz, S.R.; Moser, R.D. Fatigue behavior of Ti-6Al-4V ELI including mean stress effects. Int. J. Fatigue 2017, 99, 87-100. [CrossRef]

27. Morita, T.; Tanaka, S.; Ninomiya, S. Improvement in fatigue strength of notched Ti-6Al-4V alloy by short-time heat treatment. Mater. Sci. Eng. A 2016, 669, 127-133. [CrossRef]

28. Akahori, T.; Niinomi, M. Fracture characteristics of fatigued Ti-6Al-4V ELI as an implant material. Mater. Sci. Eng. A 1998, 243, 237-243. [CrossRef]

29. Wu, G.Q.; Shi, C.L.; Sha, W.; Sha, A.X.; Jiang, H.R. Effect of microstructure on the fatigue properties of Ti-6Al-4V titanium alloys. Mater. Des. 2013, 46, 668-674. [CrossRef]

30. Papakyriacou, M. Effects of surface treatments on high cycle corrosion fatigue of metallic implant materials. Inter. J. Fatigue 2000, 22, 873-886. [CrossRef]

31. Roach, M.D.; Williamson, R.S.; Zardiackas, L.D. Comparison of the corrosion fatigue characteristics of CP Ti-Grade 4, Ti-6Al-4V ELI, Ti-6Al-7Nb, and Ti-15Mo. J. ASTM Int. 2005, 2, 12786. [CrossRef]

32. Gao, X.-L.; Zhang, L.-J.; Liu, J.; Zhang, J.-X. Porosity and microstructure in pulsed Nd:YAG laser welded Ti6Al4V sheet. J. Mater. Process. Technol. 2014, 214, 1316-1325. [CrossRef]

33. Hong, K.-M.; Shin, Y.C. Analysis of microstructure and mechanical properties change in laser welding of Ti6Al4V with a multiphysics prediction model. J. Mater. Process. Technol. 2016, 237, 420-429. [CrossRef]

34. Xu, P.-q.; Li, L.; Zhang, C. Microstructure characterization of laser welded Ti-6Al-4V fusion zones. Mater. Charact. 2014, 87, 179-185. [CrossRef]

35. Factory certification of RTI International Metals Inc., Ingot-No.: 9711830. 
36. Shanjin, L.; Yang, W. An investigation of pulsed laser cutting of titanium alloy sheet. Opt. Lasers Eng. 2006, 44, 1067-1077. [CrossRef]

37. Rowe, W.B. Principles of Modern Grinding Technology; William Andrew: Oxford, UK, 2009.

38. Yang, S.; Li, W. Surface Finishing Theory and New Technology; Springer: Berlin, Germany, 2018.

39. Schijve, J. Fatigue of Structures and Materials; Springer: Dordrecht, The Netherlands, 2009. 